\title{
Endometriosis is the independent prognostic factor for survival in Chinese patients with epithelial ovarian carcinoma
}

\author{
Tong Ren ${ }^{1+}$, Shu Wang ${ }^{1{ }^{*}}$, Jian Sun ${ }^{2}$, Ji-Min Qu ${ }^{3}$, Yang Xiang ${ }^{1}$, Keng Shen ${ }^{1}$ and Jing He Lang ${ }^{1 *}$
}

\begin{abstract}
Background: Clinico-pathological characteristics and possible prognostic factors among women with epithelial ovarian carcinoma (EOC) with or without concurrent endometriosis were explored.

Method: We retrospectively identified 304 patients with EOC treated primarily at Peking Union Medical College Hospital with median follow-up time of 60 months.

Results: Of 304 patients with EOC, concurrent endometriosis was identified in 69 (22.7\%). The patients with concurrent endometriosis were younger and more probably post-menopausal at onset, were less likely to have abdominal distension, with significantly lower level of pre-surgery serum Ca125 and less possibility of having the history of tubal ligation. The women with concurrent endometriosis group were more likely to have early stage tumors $(88.41 \%$ versus $52.77 \%)$, receive optimal cytoreductive surgery $(92.75 \%$ versus $71.06 \%)$, and less likely to have lymph node metastasis or to develop platinum resistance disease (7.25\% versus $14.89 \%$, and $7.35 \%$ versus $20 \%)$, when compared with women without coexisting endometriosis. The univariate analysis showed that concurrent endometriosis was a prognostic factor for overall survival (OS) and disease-free survival (DFS), but this association just remained in the DFS by multivariate analysis. Besides, multivariate analysis also showed that FIGO stage, residual disease, chemotherapy cycles, chemotherapy resistance and concomitant hypertension were the independent impact factors of OS for EOC patients; whereas FIGO stage, lymphadenectomy, residual disease, coexisting endometriosis and chemoresistance were independent impact factors of DFS for those patients.
\end{abstract}

Conclusions: EOC patients with concurrent endometriosis showed distinct characteristics and had longer overall survival and disease-free survival when compared with those without endometriosis. Endometriosis was the independent prognostic factor for DFS for patients in this series.

Keywords: Endometriosis, Epithelial ovarian carcinoma, Prognostic factor

\section{Background}

Endometriosis (EM) is one of the most common benign gynecological diseases, with an incidence as high as 10$15 \%$ in women of childbearing age. EM typically manifests as masses, pain, and infertility in clinical practice, seriously affecting physical and psychological health as well as the quality of life of women of childbearing age. Nonetheless,

\footnotetext{
* Correspondence: wangshu219@hotmail.com; langjh@hotmail.com ${ }^{\dagger}$ Equal contributors

'Department of Obstetrics and Gynecology, Peking Union Medical College Hospital, Peking Union Medical College \& Chinese Academy of Medical Sciences, Shuaifuyuan 1, Wangfujing, Dongcheng District, Beijing 100730, People's Republic of China

Full list of author information is available at the end of the article
}

attention has long been paid to the malignant potential of EM $[1,2]$. In recent years, the association between malignant transformation of $\mathrm{EM}$ and the development of particular types of epithelial ovarian cancer (EOC) has become a hot topic in basic clinical research [3-6]. EM may represent a precancerous lesion of ovarian clear cell carcinoma (OCCC) or ovarian endometrioid carcinoma (OEC). As a potential "precancerous state", EM is closely associated with OCCC and OEC [7-9]. Endometriosisassociated ovarian carcinoma(EAOC) may be a special type of $\mathrm{OC}$ that shows different clinicopathological and prognostic features compared with ordinary OC $[10,11]$. The majority of existing clinical case studies have involved a 
Table 1 Clinical and morphological characteristics of EOC patients with or without EM

\begin{tabular}{|c|c|c|c|c|c|c|c|}
\hline & NON-EAOC & EAOC & P & & NON-EAOC & EAOC & P \\
\hline Numbers & 236 & 68 & & Unilateral & $161(68.51 \%)$ & $51(73.91 \%)$ & \multirow{4}{*}{0.1204} \\
\hline \multirow[t]{2}{*}{ Age(year)(median[IQR]) } & \multirow{2}{*}{$\begin{array}{l}52.0 \\
{[44.00,62.00]}\end{array}$} & \multirow{2}{*}{$\begin{array}{l}45.00 \\
{[40.00,49.50]}\end{array}$} & $<0.001^{\mathrm{a}}$ & Bilateral & 74 (31.49\%) & $18(26.09 \%)$ & \\
\hline & & & \multirow{2}{*}{$<.0001^{a}$} & Breast cancer history & & & \\
\hline Menopause & & & & Yes & $8(3.4 \%)$ & $0(0 \%)$ & \\
\hline Pre- & $108(45.96 \%)$ & $53(76.81 \%)$ & & No & $227(96.6 \%)$ & $69(100 \%)$ & \multirow{3}{*}{0.2433} \\
\hline Post- & $127(54.04 \%)$ & $16(23.19 \%)$ & \multirow{3}{*}{0.6506} & HT history & & & \\
\hline Gravid & & & & Yes & 45 (19.15\%) & 9 (13.04\%) & \\
\hline 0 & $26(11.06 \%)$ & $9(13.04 \%)$ & & No & $190(80.85 \%)$ & 60 (86.96\%) & \multirow{3}{*}{0.2263} \\
\hline$>=1$ & 209 (88.94\%) & $60(86.96 \%)$ & \multirow{3}{*}{0.5637} & DM history & & & \\
\hline Parity & & & & Yes & 16 (6.81\%) & $2(2.9 \%)$ & \\
\hline 0 & $41(17.45 \%)$ & $10(14.49 \%)$ & & No & $219(93.19 \%)$ & $67(97.1 \%)$ & \\
\hline$>=1$ & $194(82.55 \%)$ & $59(85.51 \%)$ & \multirow{4}{*}{0.3989} & Hysterectomy history & & & 0.1469 \\
\hline Symptoms & & & & Yes & 7 (2.98\%) & $0(0 \%)$ & \multirow{5}{*}{$0.0379^{\mathrm{a}}$} \\
\hline Abdominal pain & & & & No & $228(97.02 \%)$ & $69(100 \%)$ & \\
\hline Yes & $76(32.34 \%)$ & $17(24.64 \%)$ & & Tube ligation history & & & \\
\hline No & $158(67.23 \%)$ & $52(75.36 \%)$ & \multirow[b]{2}{*}{$0.0193^{\mathrm{a}}$} & Yes & $14(5.96 \%)$ & $0(0 \%)$ & \\
\hline Bloating & & & & No & 221 (94.04\%) & 69 (100\%) & \\
\hline
\end{tabular}

No

Palpable mass

Yes

No

Incidental finding

Yes

No

Irregular menstruation

Yes

No

Postmenopausal bleeding

Yes

No

Cachexia

Yes

No

Abnormal vaginal discharge

Yes

No

Pre-surgery Ca125 (IU/ml) (median[IQR])

Ca125 in normal range

Yes

No

Tumor size $(\mathrm{cm})$ (median[IQR])

Side of ovarian tumor
$54(22.98 \%) \quad 7(10.14 \%)$

$181(77.02 \%) \quad 62(89.86 \%)$

0.2629

$59(25.11 \%) \quad 22(31.88 \%)$

$176(74.89 \%) \quad 47(68.12 \%)$

$33(14.04 \%) \quad 15(21.74 \%)$

$202(85.96 \%) \quad 54(78.26 \%)$

0.1111

$19(8.09 \%) \quad 10(14.49 \%)$

$216(91.91 \%) \quad 59(85.51 \%)$

$18(7.66 \%) \quad 1(1.45 \%)$

$217(92.34 \%) \quad 68(98.55 \%)$

$10(4.26 \%) \quad 0(0 \%)$

$225(95.74 \%) \quad 69(100 \%)$

$2(0.85 \%) \quad 0(0 \%)$

$233(99.15 \%) \quad 69(100 \%)$

$256.20 \quad 89.50$

$[62.96,959.30] \quad[37.80,346.85]$

$<0.001^{a}$

0.2110

$39(16.6 \%) \quad 16(23.19 \%)$

$196(83.4 \%) \quad 53(76.81 \%)$

9.00

10.00

$[7.00,15.000]$

Abbreviation: $E O C$, epithelial ovarian carcinoma; $E M$, endometriosis; $E A O C$, endometriosis-associated ovarian cancer; IQR, InterQuartile Range; Ca125, cancer antigen 125; $H T$, hypertension; $D M$, diabetic mellitus;

athe difference reached statistical significance

small sample size and short follow-up time. In the present study, we compared the clinicopathological features and prognosis of OCCC and OEC with and without EM, using data from patients undergoing primary treatment for epithelial OC in our hospital.

\section{Methods}

By reviewing the medical charts, we retrospectively identi0.0610 fied 186 patients with OEC and 118 patients with OCCC who were primarily treated and received surgery at the Division of Gynecological Oncology of the Department of Obstetrics and Gynecology at Peking Union Medical College Hospital between January 2000 and January 2012. All patients received surgery and chemotherapy and were followed up at our institution. The patient follow-up period ended in March 2017. This study was approved by the University Institutional Review Board.

Here, we defined EOC with concurrent endometriosis as the presence of ovarian cancer and endometriosis identified histologically in the same ovary, the presence of endometriosis in one ovary and of ovarian cancer in the contralateral ovary, or the presence of ovarian cancer and extraovarian pelvic endometriosis (eg, peritoneal endometriosis). According to the pathological criteria listed above, we identified 68 of patients with ovarian carcinoma with concurrent EM (EAOC group), The remaining 236 patients had no pathological evidence of endometriosis (non-EAOC group). 
The collected clinico-pathological data were compared between two groups as shown in Table 1. The 304 patients were subjected to surgical-pathologic restaging based on the International Federation of Gynecology and Obstetrics (FIGO) staging guidelines for ovarian cancer (2013) [12]. For statistical analysis, FIGO stage categories were classified into early stage (FIGO stages I and II subjects) and late stage (FIGO stages III to IV).

Besides, synchronous tumors of the ovary and endometrium were found and analyzed in this series. The criteria of Young and Scully [13] was used for interpretation of synchronous primary tumors of both organs or of metastasis from one organ to the other. When the pathologic study reveals similar types, the differentiation between the 2 separate primary cancers or 1 single advanced cancer with metastasis is much more difficult. Herein, we apply standardized criteria to differentiate the 2 synchronous cancers, rather than 1 cancer metastases [(1) both tumors are confined to primary sites; (2) no direct extension between the tumors; (3) no lymphovascular tumor emboli; (4) no or only superficial myometrial invasion of the endometrial lesion; and (5) no distant metastasis] [14, 15].

Disease-free survival (DFS) was defined as the time interval from the date of primary surgery to the date of disease progression and/or recurrence. Overall survival (OS) was defined in months as the date of the primary surgery to the date of death or censoring at the date of last contact.

Continuous variables were analyzed using Mann-Whitney $\mathrm{U}$ test. Categorical variables were analyzed by using test or Fisher's exact test. Receiver Operating Characteristic (ROC) curve was constructed to define the optimal cutoff value for stratifying and grouping continuous variables. Survival comparisons were obtained using the log-rank test in an unadjusted Kaplan-Meier model. Cox proportional hazards regression was used for multivariate analysis. Variables included in this analysis were those found to be statistically significant in the univariate analyses. Hazards ratios and 95\% confidence intervals (CIs) were used to calculate the relative risk of death or relapse for each variable of interest while adjusting for other covariates. All $P$ values reported were 2 tailed, and a $P$ value of 0.05 or less was considered as statistical significance.

\section{Results}

Table 1 shows the statistical results for the clinical information from the two groups of patients. The patients in EAOC group exhibited an median age of onset of 45 years, which was 7 years younger than that of the patients in non-EAOC group; this between-group difference was statistically significant $(P<0.001)$. Compared with the EAOC patients, a higher proportion of non-EAOC patients were post-menopausal at onset $(P<0.001)$. No significant difference in the number of pregnancies was found between the two groups. The leading three most common referral symptoms for women in EAOC group were palpable mass $(31.88 \%)$, abdominal pain $(24.64 \%)$ and incidental finding(21.74\%), while which for ones in Non-EAOC group were respectively abdominal pain(32.34\%), palpable

Table 2 The surgico-pathological characters and treatment-related variables of EOC patients with or without EM

\begin{tabular}{|c|c|c|c|}
\hline & NON-EAOC & EAOC & $P$ \\
\hline FIGO Stage ${ }^{a}$ & & & $<.0001^{*}$ \\
\hline 1 & $92(39.15 \%)$ & $49(71.01 \%)$ & \\
\hline$\|$ & $32(13.62 \%)$ & $12(17.39 \%)$ & \\
\hline III & $97(41.28 \%)$ & $7(10.14 \%)$ & \\
\hline IV & $14(5.96 \%)$ & $1(1.45 \%)$ & \\
\hline Early or late Stage & & & $<.0001^{*}$ \\
\hline $1+\|$ & $124(52.77 \%)$ & $61(88.41 \%)$ & \\
\hline$I I I+I V$ & $111(47.23 \%)$ & $8(11.59 \%)$ & \\
\hline Lymphadenectomy & & & $0.0160^{*}$ \\
\hline Yes & 189 (80.43\%) & $64(92.75 \%)$ & \\
\hline No & $46(19.57 \%)$ & $5(7.25 \%)$ & \\
\hline Residual disease & & & $0.0002^{*}$ \\
\hline No or $<1 \mathrm{~cm}$ & $167(71.06 \%)$ & $64(92.75 \%)$ & \\
\hline$>1 \mathrm{~cm}$ & 68 (28.94\%) & $5(7.25 \%)$ & \\
\hline Metastasis of lymph node & & & $0.0060^{*}$ \\
\hline Yes & 35 (14.89\%) & $5(7.25 \%)$ & \\
\hline No & $154(65.53 \%)$ & $59(85.51 \%)$ & \\
\hline Not-dissected & $46(19.57 \%)$ & $5(7.25 \%)$ & \\
\hline Histotype & & & $0.0041^{*}$ \\
\hline Clear cell & $81(34.47 \%)$ & $37(53.62 \%)$ & \\
\hline Endometrioid & $154(65.53 \%)$ & $32(46.38 \%)$ & \\
\hline Endometrial disorders & & & $0.0808^{*}$ \\
\hline Yes & $45(19.15 \%)$ & $7(10.14 \%)$ & \\
\hline No & 190 (80.85\%) & $62(89.86 \%)$ & \\
\hline Endometrial cancer & & & 0.051 \\
\hline Yes & $25(23.8 \%)$ & $2(7.4 \%)$ & \\
\hline No & $211(76.2 \%)$ & $66(92.6 \%)$ & \\
\hline Chemotherapy & & & 0.6250 \\
\hline Platinum-based & $224(95.32 \%)$ & $67(97.1 \%)$ & \\
\hline Other regimen & $3(1.28 \%)$ & $0(0 \%)$ & \\
\hline No chemotherapy & $8(3.4 \%)$ & $2(2.9 \%)$ & \\
\hline Chemo Cycles & $6.36 \pm 2.33$ & $5.70 \pm 2.05$ & $0.039^{*}$ \\
\hline Chemo-resistance & & & $0.0149^{*}$ \\
\hline Yes & 47 (20\%) & $5(7.35 \%)$ & \\
\hline No & 188 (80\%) & $63(92.65 \%)$ & \\
\hline
\end{tabular}

Abbreviation: $E O C$, epithelial ovarian carcinoma; FIGO, International Federation of Gynecology and Obstetrics; $E M$, endometriosis; $E A O C$, endometriosis-associated ovarian cancer

*the difference reached statistical significance

according to the classification system of FIGO staging (2013 version) 
mass (25.11\%), bloating (22.98\%). Bloating was the symptom in a greater proportion of patients in non-EAOC group compared with ones in EAOC group $(P=0.0193)$, whereas no significant differences in other symptoms were found between the two groups.

The patients of EAOC group showed significantly lower preoperative CA125 levels than non-EAOC group (median[IQR], 89.50[37.80, 346.85] $\mathrm{mIU} / \mathrm{ml}$ vs 256.20[62.96, 959.30] $\mathrm{mIU} / \mathrm{ml}, P<0.001)$. But the proportion of patients presented CA125 levels within the normal range was not significantly different $(23.19 \%$ vs. $16.6 \%, P=0.2110)$. No significant differences were found between the two groups in terms of tumor size or tumor side, and neither difference seen in the medical history or complication of breast cancer, hypertension (HT), diabetic mellitus (DM) and previous surgery of hysterectomy. However, there was a significant difference between the two groups in terms of tubal ligation (non-EAOC vs EAOC, 5.96\% vs $0 \%, P=0.0379$ ).
Table 2 summarizes the surgico-pathological data. In EAOC group, $71.01 \%$ of patients were at FIGO stage I, while $88.41 \%$ had early (stage I + II) cancer. In nonEAOC group, $47.23 \%$ of the patients displayed advanced cancer. The between-group difference in tumor stage reached statistical significance $(P<0.001)$. The rate of performing lymphadenectomy in EAOC group was significantly higher than in non-EAOC group $(92.75 \%$ vs $80.43 \%, P=0.0160$ ). Among the 68 patients in EAOC group, OCCC accounted for $53.62 \%$, OEC for $46.38 \%$; the non-EAOC patients mainly had OEC; these two types of cancer accounted for $34.47 \%$ and $65.53 \%$ of the patients, respectively $(P=0.0041)$. Besides, the rate of having concurrent endometrial disorders between two groups was similar $(P=0.0808)$, but the rate of concurrent endometrial carcinoma in non-EAOC group was higher than EAOC group ( $23.8 \%$ vs $7.4 \%, P=0.051)$.

The proportion of patients who received postoperative chemotherapy and platinum-based chemotherapy between

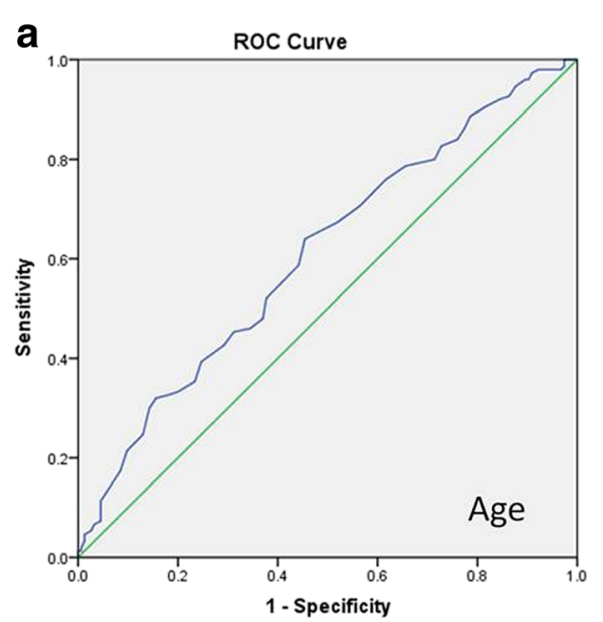

Diagonal segments are produced by ties b

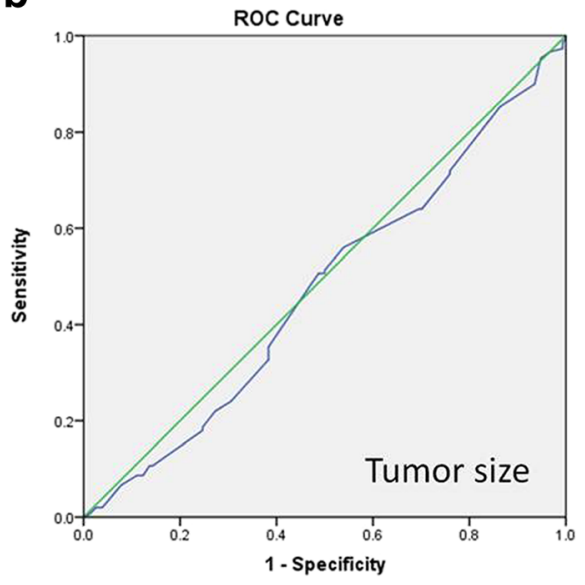

Diagonal segments are produced by ties.

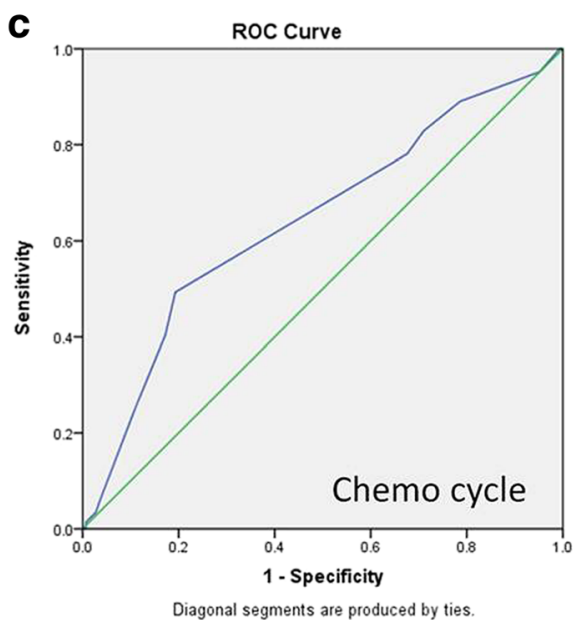

Fig. 1 a ROC curve for age of disease onset in DFS; b. ROC curve for tumor size in DFS; c. ROC curse for cycles of platinum-based chemotherapy in DFS 
Table 3 Univariate analysis of overall survival among EOC patients

\begin{tabular}{|c|c|c|c|c|c|}
\hline Variable & Category & $N(\%)$ & OS (Median[IQR]) & Survival rate(\%) & $P$ \\
\hline \multirow[t]{2}{*}{ Age y } & $<49$ & $138(45.39)$ & $69.5[38,102]$ & 84.06 & $0.0013^{*}$ \\
\hline & $>=49$ & $166(54.61)$ & $53[23,78]$ & 71.08 & \\
\hline \multirow[t]{2}{*}{ Menopausal status } & Pre & $161(52.96)$ & $68[38,102]$ & 81.99 & $0.0043^{*}$ \\
\hline & Post & $143(47.04)$ & $53[22,77]$ & 71.33 & \\
\hline \multirow[t]{2}{*}{ Gravidity } & 0 & $35(11.51)$ & $60[34,107]$ & 82.86 & 0.3070 \\
\hline & $>=1$ & 269 (88.49) & $60[27,89]$ & 75.84 & \\
\hline \multirow[t]{2}{*}{ Parity } & 0 & $51(16.78)$ & $70[40,96]$ & 84.31 & 0.1016 \\
\hline & $>=1$ & 253 (83.22) & $58[26,86]$ & 75.1 & \\
\hline \multirow[t]{2}{*}{ Stage } & Early & $185(60.86)$ & $69[47,102]$ & 91.35 & $<.0001^{*}$ \\
\hline & Late & 119 (39.14) & $38[18,72]$ & 53.78 & \\
\hline \multirow[t]{4}{*}{ FIGO Stage } & । & $141(46.38)$ & $72[51,102]$ & 95.74 & $<.0001^{*}$ \\
\hline & $\|$ & $44(14.47)$ & $60[33,110]$ & 79.55 & \\
\hline & III & $104(34.21)$ & $41.5[20,73.5]$ & 53.85 & \\
\hline & IV & $15(4.93)$ & $10[5,53]$ & 53.33 & \\
\hline \multirow[t]{2}{*}{ Histotype } & Clear cell & $118(38.82)$ & $58.5[27,88]$ & 78.81 & 0.6479 \\
\hline & Endometrioid & $186(61.18)$ & $60.5[31,92]$ & 75.81 & \\
\hline \multirow[t]{2}{*}{ Lymphadenectomy } & Yes & $51(16.78)$ & $38[12,69]$ & 52.94 & $<.0001^{*}$ \\
\hline & No & 253 (83.22) & $62[34,91]$ & 81.82 & \\
\hline \multirow[t]{2}{*}{ Metastasis of LN } & Positive & $40(13.16)$ & $32.5[15,59]$ & 57.5 & $<.0001^{*}$ \\
\hline & Negative & $213(70.07)$ & $68[44,101]$ & 86.38 & \\
\hline \multirow[t]{2}{*}{ Residual disease } & $>1 \mathrm{~cm}$ & $73(24.01)$ & $33[15,65]$ & 50.68 & $<.0001^{*}$ \\
\hline & None or $<1 \mathrm{~cm}$ & $231(75.99)$ & $66[38,98]$ & 85.28 & \\
\hline \multirow[t]{2}{*}{ CA125 normal } & Yes & 55 (18.09) & $73[52,102]$ & 92.73 & $0.0009^{*}$ \\
\hline & No & $249(81.91)$ & $58[24,87]$ & 73.49 & \\
\hline \multirow[t]{2}{*}{ Tumor size(cm) } & $<10$ & $153(50.33)$ & $60[32,90]$ & 77.12 & 0.8933 \\
\hline & $\geq 10$ & $151(49.67)$ & $60[26,88]$ & 76.82 & \\
\hline \multirow[t]{2}{*}{ Tumor side } & Unilateral & $212(69.74)$ & $61[33.5,96.5]$ & 80.66 & $0.0063^{*}$ \\
\hline & Bilateral & $92(30.26)$ & $53[24,84.5]$ & 67.39 & \\
\hline \multirow[t]{2}{*}{ Endometriosis } & No & $235(77.30)$ & $57[24,88]$ & 72.77 & $0.0010^{*}$ \\
\hline & Yes & $69(22.70)$ & $67[42,101]$ & 91.3 & \\
\hline \multirow[t]{2}{*}{ Endometrial disorders } & No & $252(82.89)$ & $60[29.5,91]$ & 75.0 & 0.2184 \\
\hline & Yes & $52(17.11)$ & $60[29,85.5]$ & 84.62 & \\
\hline \multirow[t]{2}{*}{ Endometrial cancer } & No & 252 (82.89) & $60[26.5,91]$ & 74.6 & 0.2321 \\
\hline & Yes & $27(8.88)$ & $60[40,96]$ & 85.19 & \\
\hline \multirow[t]{2}{*}{ Hypertension } & No & $250(82.24)$ & $60[32,92]$ & 79.6 & $0.0132^{*}$ \\
\hline & Yes & $54(17.76)$ & $57.5[19,81]$ & 64.81 & \\
\hline \multirow[t]{2}{*}{ Chemotherapy cycle } & $<4$ & $191(62.83)$ & $61[34,91]$ & 80.1 & $0.0373^{*}$ \\
\hline & $\geq 4$ & $100(32.89)$ & $53[23.5,85]$ & 70 & \\
\hline \multirow[t]{2}{*}{ Chemo-resistance } & No & 252 (82.89) & $67[44,99.5]$ & 84.52 & $<.0001^{*}$ \\
\hline & Yes & $52(17.11)$ & $19[11,28.5]$ & 40.38 & \\
\hline
\end{tabular}

$P$ values were cultivated by Kaplan-Meier analysis

two groups were likely. The mean cycles $( \pm S D)$ of platinumbased chemotherapy were significantly divergent between two groups $(6.36 \pm 2.33$ in non-EAOC vs $5.70 \pm 2.05$ in
EAOC, $P=0.039)$. We defined chemo-resistance as tumor recurrence or progression within 6 months after the last chemotherapy. In this study, the rate of 
Table 4 Univariate analysis of disease-free survival among patients

\begin{tabular}{|c|c|c|c|c|c|}
\hline Variable & Category & $N(\%)$ & OS (Median(IQR)) & Survival rate(\%) & $P$ \\
\hline \multirow[t]{2}{*}{ Age y } & $<49$ & $141(46.38)$ & $52[16,87]$ & 57.45 & $0.0194^{\mathrm{a}}$ \\
\hline & $>=49$ & $163(53.62)$ & $31[12,65]$ & 47.85 & \\
\hline \multirow[t]{2}{*}{ Menopausal status } & Pre & $161(52.96)$ & $50[17,87]$ & 57.14 & $0.0052^{a}$ \\
\hline & Post & $143(47.04)$ & $26[11,64]$ & 46.85 & \\
\hline \multirow[t]{2}{*}{ Gravidity } & 0 & $35(11.51)$ & $44[16,90]$ & 51.43 & 0.6329 \\
\hline & $>=1$ & 269 (88.49) & $39[12,75]$ & 52.79 & \\
\hline \multirow[t]{2}{*}{ Parity } & 0 & $51(16.78)$ & $51[15,86]$ & 52.94 & 0.5262 \\
\hline & $>=1$ & 253 (83.22) & $38[12,75]$ & 52.57 & \\
\hline \multirow[t]{2}{*}{ Stage } & Early & $185(60.86)$ & $59[36,88]$ & 74.59 & $<.0001^{\mathrm{a}}$ \\
\hline & Late & 119 (39.14) & $14[8,28]$ & 18.49 & \\
\hline \multirow[t]{4}{*}{ FIGO Stage } & । & $141(46.38)$ & $62[44,90]$ & 81.56 & $<.0001^{\mathrm{a}}$ \\
\hline & $\|$ & $44(14.47)$ & $42[17.5,80]$ & 50 & \\
\hline & III & $104(34.21)$ & $15[9,30.5]$ & 20.19 & \\
\hline & IV & $15(4.93)$ & $9[0.5,22]$ & 6.67 & \\
\hline \multirow[t]{2}{*}{ Histotype } & Clear cell & $118(38.82)$ & $52.5[14,85]$ & 61.86 & $0.0091^{\mathrm{a}}$ \\
\hline & Endometrioid & $186(61.18)$ & $34.5[12,70]$ & 46.24 & \\
\hline \multirow[t]{2}{*}{ Lymphadenectomy } & Yes & $51(16.77)$ & $12[6,26]$ & 23.53 & $<.0001^{\mathrm{a}}$ \\
\hline & No & 253 (83.22) & $48[17,82]$ & 58.1 & \\
\hline \multirow[t]{2}{*}{ Metastasis of LN } & Positive & $213(70.07)$ & $54[24,86]$ & 64.79 & $<.0001^{\mathrm{a}}$ \\
\hline & Negative & $40(13.16)$ & $13[7,31]$ & 22.5 & \\
\hline \multirow[t]{2}{*}{ Residual disease } & $>1 \mathrm{~cm}$ & 231 (75.99) & $53[22,84]$ & 17.81 & $<.0001^{a}$ \\
\hline & None or $<1 \mathrm{~cm}$ & $73(24.01)$ & $12[5,23]$ & 63.2 & \\
\hline \multirow[t]{2}{*}{ CA125 normal } & Yes & $55(18.09)$ & $64[48,97]$ & 74.55 & $<.0001^{\mathrm{a}}$ \\
\hline & No & 249 (81.91) & $30[12,70]$ & 47.39 & \\
\hline \multirow[t]{2}{*}{ Tumor size(cm) } & $<10$ & $153(50.33)$ & $40[13,77]$ & 50.33 & 0.7834 \\
\hline & $\geq 10$ & $151(49.67)$ & $38[13,75]$ & 54.3 & \\
\hline \multirow[t]{2}{*}{ Tumor side } & Unilateral & $212(69.74)$ & $50.5[14.5,83]$ & 60.85 & $<.0001^{\mathrm{a}}$ \\
\hline & Bilateral & $92(30.26)$ & $27.5[12,55.5]$ & 33.7 & \\
\hline \multirow[t]{2}{*}{ Endometriosis } & No & $235(77.30)$ & $31[12,69]$ & 44.68 & $<.0001^{\mathrm{a}}$ \\
\hline & Yes & $69(22.70)$ & $60[36,89]$ & 78.26 & \\
\hline \multirow[t]{2}{*}{ Endometrial disorders } & No & $252(82.89)$ & $38[13,75.5]$ & 51.19 & 0.3357 \\
\hline & Yes & $52(17.11)$ & $48[13,76]$ & 57.69 & \\
\hline \multirow[t]{2}{*}{ Endometrial cancer } & No & $252(82.89)$ & $38[13,75.5]$ & 51.19 & 0.2503 \\
\hline & Yes & $27(8.88)$ & $49[18,86]$ & 59.26 & \\
\hline \multirow[t]{2}{*}{ Hypertension } & No & $250(82.24)$ & $41[14,76]$ & 52.8 & 0.4164 \\
\hline & Yes & $54(17.763)$ & $29.5[11,68]$ & 50 & \\
\hline \multirow[t]{2}{*}{ Chemotherapy cycle } & $<4$ & $191(62.83)$ & $51[19,83]$ & 61.78 & $<.0001^{\mathrm{a}}$ \\
\hline & $\geq 4$ & 100 (32.89) & $19[11,45]$ & 32 & \\
\hline \multirow[t]{2}{*}{ Chemotherapy resistence } & No & $252(82.89)$ & $51.5[24,84]$ & 63.1 & $<.0001^{\mathrm{a}}$ \\
\hline & Yes & 52 (17.11) & $7.5[3,10]$ & 0 & \\
\hline
\end{tabular}

Abbreviation: EOC, epithelial ovarian carcinoma; FIGO, International Federation of Gynecology and Obstetrics

a the difference reached statistical significance. $P$ values were cultivated by Kaplan-Meier analysis 


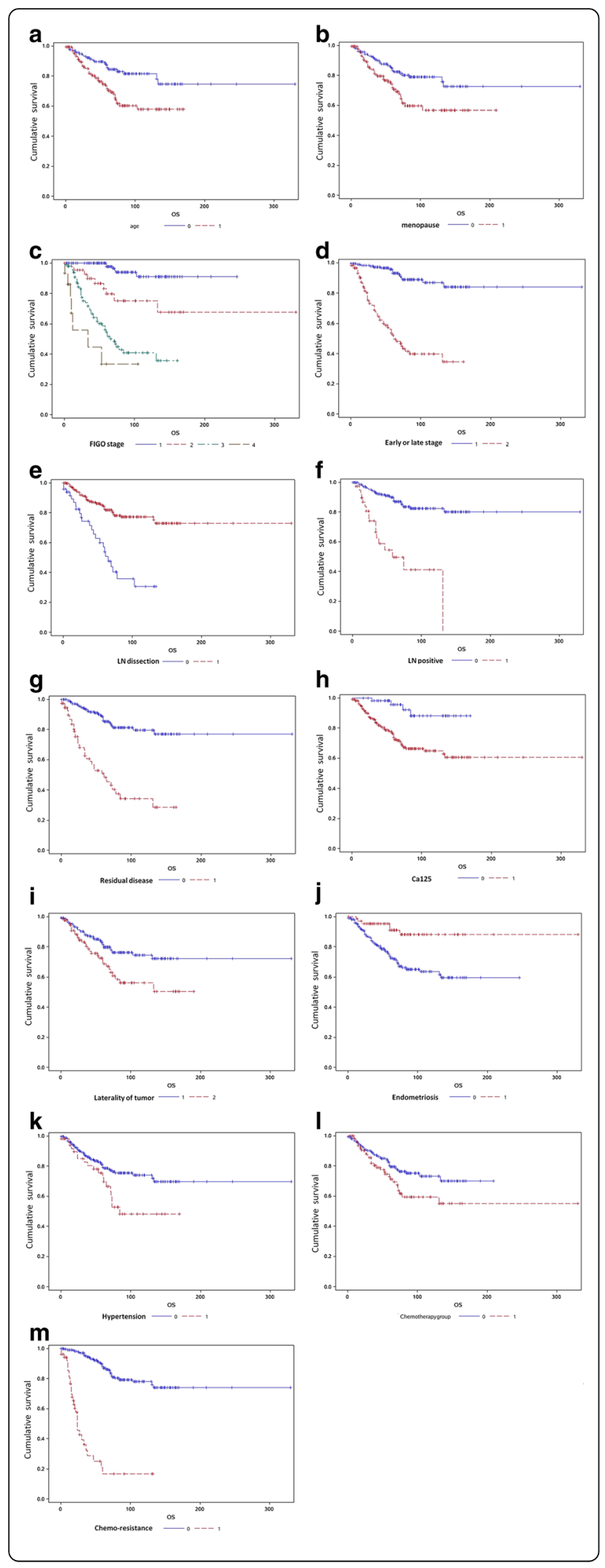

Fig. 2 A univariate analysis of overall survival in patients with EOC. Kaplan-Meier survival curves showing the effects of age (a), menopausal status (b), FIGO stage (c), early or late stage (d), lymphadenectomy (e), metastasis of LN (f), residual disease $(\mathbf{g})$, normal level of Ca125(h), unilateral or bilateral tumor (i), endometriosis $(\mathbf{j})$, hypertension $(\mathbf{k})$, chemotherapy courses (I) and chemo-resistance (m) for OS

occurring chemo-resistance in non-EAOC group was significantly higher than in non-EAOC group $(20 \%$ vs $7.35 \%, P=0.0149$ ).

With an median(IQR) follow-up of $60[29,90]$ months, the entire study cohort observed $23.03 \%$ of deaths due to disease, and $47.37 \%$ of patients developed relapse disease with the median(IQR) time of DFS of 39[13,76] months.

Receiver Operating Characteristic (ROC) curve constructed to stratify the continuous variable including the age of onset, tumor size, and the courses of platinum regimen received. As a result, the optimal cutoff value was defined as 49 years for age, $10 \mathrm{~cm}$ for tumor size, 3 for chemotherapy courses (Showed in Fig. 1).

As seen in Tables 3 and 4, the univariate analysis showed that concurrent endometriosis was a prognostic factor for overall survival (OS, $P=0.0010)$ and disease-free survival (DFS, $P<0.0001$ ). Besides, the following variables were showed relating to either OS or PFS, including age $(P=0.0013, P=0.0194)$, menopausal status $(P=0.0043$, $P=0.0052)$, FIGO stage $(P<0.0001, P<0.0001)$, early or late stage $(P<0.0001, P<0.0001)$, lymphadenectomy $(P<0.0001, P<0.0001)$, metastasis of LN $(P<0.0001$, $P<0.0001)$, residual disease $(P<0.0001, P<0.0001)$, normal level of Ca125 $(P=0.0009, P<0.0001)$, unilateral or bilateral tumor $(P=0.0063, P<0.0001)$, chemotherapy courses $(P=0.0373, P<0.0001)$ and chemo-resistance $(P<0.0001$, $P<0.0001$ ) (showed in Fig. 2). And the correlation was also seeen in OS and hypertension, but not for PFS (showed in Fig. 3).

Multivariate analysis showed coexisting endometriosis was the independent risk factor of DFS for this series of patients $(P=0.0233)$, but not OS $(P=0.4038)$. And the Tables 5 and 6 also revealed that FIGO stage $(P=0.0001)$, chemotherapy cycles $(P=0.0058)$, chemotherapy resistance $(P<0.0001)$ and concomitant hypertension $(P=0.0102)$ were the independent impact factors of OS, whereas FIGO stage $(P<0.0001)$, lymphadenectomy $(P=0.0084)$, residual disease $(P=0.0165)$, and chemoresistance $(P<0.0001)$ were independent impact factors of DFS for EOC patients in this study.

\section{Discussion}

In this study, 68 of 304 patients enrolled exhibited an association with EM, which accounted for $22.37 \%$ of the patients with EOC. The incidence of EAOC in our patients was close to the values reported by Nezhat [16]. 


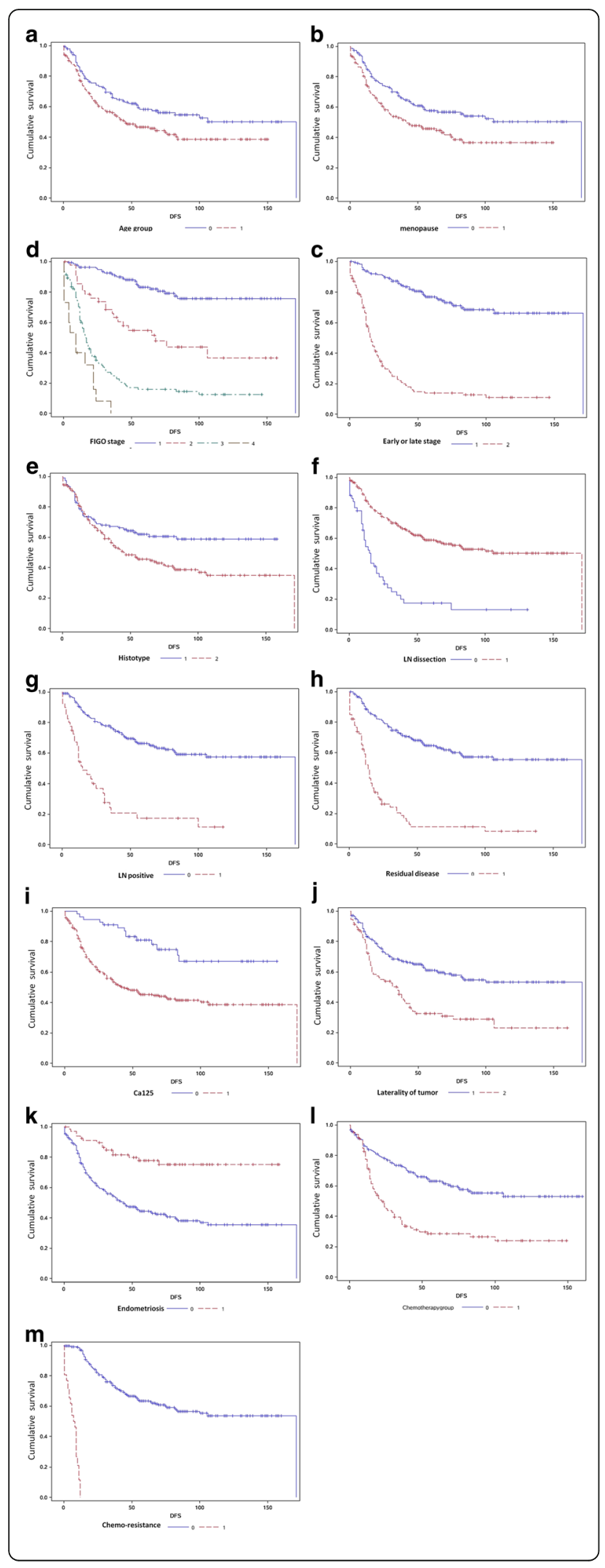

Fig. 3 A univariate analysis of disease-free survival in patients with ovarian clear cell and endometrioid carcinoma. Kaplan-Meier survival curves showing the effects of age (a), menopausal status (b), FIGO stage $(\mathbf{c})$, early or late stage $(\mathbf{d})$, histotype (e), lymphadenectomy $(\mathbf{f})$, metastasis of $\mathrm{LN}(\mathbf{g})$, residual disease $(\mathbf{h})$, Ca125 in normal range (i), unilateral or bilateral tumor (j), endometriosis (k), chemotherapy courses (I) and chemo-resistance $(\mathbf{m})$ for DFS

With respect to clinical features, we found that the patients in EAOC group exhibited a markedly lower median age of onset than the non-EAOC patients (45 years vs. 52 years); most EAOC patients were premenopausal at onset $(76.81 \%$ vs. $45.96 \%)$. This is in agreement with the results of Garrett [17], Lim [18], and Scarfone [19]. We wonder if this between-group difference may be due to the following factors: the EAOC patients presented more evident clinical symptoms and tended to undergo pelvic examination more frequently, indirectly moving the timing of the detection of ovarian tumors forward. However, in the present study, we found no significant difference in clinical symptoms between the two groups, except that a relatively high proportion of non-EAOC patients exhibited bloating as the first symptom $(22.98 \%$ vs. $10.14 \%)$. From this aspect, We tend to believe that EAOC shows a younger age of onset due to intrinsic mechanisms, rather than simply early detection $[9,10]$.

Median preoperative serum CA125 levels were significantly different between the two groups of patients (256.20 vs. $89.50 \mathrm{U} / \mathrm{ml}$ ), a finding that is inconsistent with the results of Scarfone [19] and Mangili [20]. It is currently thought that CA125, a predictor of malignant ovarian tumors, is divergent among the different histological type of tumors [21, 22]. Therefore, precise classification of epithelial OC, followed by the identification of new tumor markers, will have important clinical value, and further relevant research is expected to be conducted.

Comparison of surgical histories between groups revealed that a markedly higher proportion of non-EAOC patients relative to EAOC patients underwent tubal ligation, but no difference for hysterectomy. Van Gorp [23], Nagle [24], and Rice [25, 26] have shown that hysterectomy or tubal ligation can decrease the incidence of EM and EAOC to varying degrees, which may explain our results. That is, tubal ligation may have reduced the development of EAOC in this series of patients. This result is interesting and remains to be verified in a largesample, prospective, long-term study.

Similar to the results of numerous studies [18-20], we found that most OCCC and OEC patients of the EAOC group (71.01\%) had FIGO I stage cancer at diagnosis. A markedly higher proportion of patients had early (stage I-II) cancer in the EAOC group compared with the non-EAOC group $(88.41 \%$ vs. $52.77 \%)$. It is therefore easier to perform lymphadenectomy and to achieve 
Table 5 Multivariable analysis of overall survival by Cox regression model for EOC patients

\begin{tabular}{|c|c|c|c|c|c|}
\hline Variable & $\beta$ & Standard Error & Chi-Square & $P$ & $\mathrm{HR}(95 \% \mathrm{Cl})$ \\
\hline Age group & -0.72 & 0.43 & 2.83 & 0.0923 & $0.49(0.21 \sim 1.13)$ \\
\hline Menopause & 0.76 & 0.44 & 3.00 & 0.0835 & $2.14(0.9 \sim 5.06)$ \\
\hline Stage (early or late) & 1.48 & 0.39 & 14.63 & $0.0001^{*}$ & $4.40(2.06 \sim 9.4)$ \\
\hline Histotype & -0.36 & 0.28 & 1.69 & 0.1941 & $0.7(0.4 \sim 1.2)$ \\
\hline Lymph node positive & 0.11 & 0.38 & 0.09 & 0.7667 & $1.12(0.53 \sim 2.36)$ \\
\hline Lymphadenectomy & 0.43 & 0.33 & 1.73 & 0.1887 & $1.54(0.81 \sim 2.95)$ \\
\hline Residual disease & 0.51 & 0.28 & 3.47 & 0.0625 & $1.67(0.97 \sim 2.87)$ \\
\hline CA125 normal & 0.68 & 0.56 & 1.51 & 0.219 & $1.98(0.67 \sim 5.89)$ \\
\hline Tumor side & 0.29 & 0.27 & 1.17 & 0.2797 & $1.34(0.79 \sim 2.28)$ \\
\hline Endometriosis & -0.39 & 0.47 & 0.70 & 0.4038 & $0.68(0.27 \sim 1.69)$ \\
\hline Chemotherapy course & -0.78 & 0.28 & 7.61 & $0.0058^{*}$ & $0.46(0.26 \sim 0.8)$ \\
\hline Chemoresistance & 1.94 & 0.32 & 37.68 & $<.0001^{*}$ & $6.96(3.75 \sim 12.93)$ \\
\hline Hypertension & 0.77 & 0.30 & 6.61 & $0.0102^{*}$ & $2.16(1.2 \sim 3.89)$ \\
\hline
\end{tabular}

*the difference reached statistical significance. $P$ values were cultivated by Cox regression analysis. The overall test of the above model showed the model was significance, $p<0.0001$

satisfying debulking surgery in the former group than in the latter group (respectively $92.75 \%$ vs $80.43 \%$ and $92.75 \%$ vs $71.06 \%$ ). As discussed previously, it is unclear whether the earlier clinicopathological stage of EAOC patients is due to early detection or the biological behavior of the tumor. Currently, there is no evidence showing that this patient population exhibits particular first symptoms or a specific medical history leading to early detection of tumors. Therefore, we tend to believe that EAOC may possess certain unique cellular and molecular biological characteristics that result in slow progression of the disease [10].

Although the cases enrolled in this study spanned a period of more than 12 years, during which there was no major revision in the chemotherapy regimens and strategies for treating OC. We evaluated the situation of postoperative chemotherapy regimens patients had received. Most patients underwent postoperative platinumbased chemotherapy in the two groups (92.9\% vs. $95.8 \%)$. The results showed no significant difference between the two groups with regard to receiving postoperative adjuvant chemotherapy. Nevertheless, EAOC patients received less courses of chemotherapy(mean \pm SD, $5.70 \pm 2.05$ vs $6.36 \pm 2.33$ cycles) and a markedly lower percentage of EAOC patients received were platinum resistant, when compared with non-EAOC patients (7.35\% versus $20 \%$ ). The findings suggest that EAOC may involve specific molecular mechanisms, which are partially reflected in the sensitivity to chemotherapeutic drugs and must be further investigated.

Table 6 Multivariable analysis of disease-free survival by Cox regression model for EOC patients

\begin{tabular}{llllll}
\hline Variable & $\beta$ & Standard Error & Chi-Square & $P$ & HR(95\% Cl) \\
\hline Age group & 0.13 & 0.30 & 0.19 & 0.6653 & $1.14(0.63 \sim 2.07)$ \\
Menopause & 0.14 & 0.29 & 0.22 & 0.6377 & $1.15(0.65 \sim 2.03)$ \\
Stage (early or late) & 1.03 & 0.26 & 15.78 & $<.0001^{*}$ & $2.79(1.68 \sim 4.64)$ \\
Histotype & 0.26 & 0.19 & 1.87 & 0.1713 & $1.3(0.89 \sim 1.9)$ \\
Lymph node positive & 0.22 & 0.24 & 0.84 & 0.3605 & $1.25(0.77 \sim 2.02)$ \\
Lymphadenectomy & 0.61 & 0.23 & 6.96 & $0.0084^{*}$ & $1.84(1.17 \sim 2.89)$ \\
Residual disease & 0.48 & 0.20 & 5.75 & $0.0165^{*}$ & $1.62(1.09 \sim 2.39)$ \\
CA125 normal & 0.25 & 0.31 & 0.68 & 0.4086 & $1.29(0.71 \sim 2.36)$ \\
Tumor side & 0.26 & 0.19 & 0.1619 & $1.3(0.9 \sim 1.87)$ \\
Endometriosis & -0.66 & 0.29 & 5.15 & $0.0233^{*}$ & $0.52(0.29 \sim 0.91)$ \\
Chemotherapy group & -0.03 & 0.19 & 0.02 & 0.8773 & $0.97(0.66 \sim 1.42)$ \\
Chemoresistance & 3.77 & 0.42 & 81.43 & $<.0001^{*}$ & $43.3(19.1 \sim 98.15)$ \\
\hline
\end{tabular}

*the difference reached statistical significance. $P$ values were cultivated by Cox regression analysis. The overall test of the above model showed the model was significance, $p<0.0001$ 
In this study, the median follow-up time was 60 months. Our results showed that the EAOC patients exhibited significantly longer OS and DFS time than the non-EAOC group (median[IQR], 67[42,101] vs 57[24,88]months and $60[36,89]$ vs $31[12,69]$ months, as seen in Tables 3 and 4). Different results have been reported previously. A metaanalysis conducted by Kim et al. showed a better OS rate for EAOC patients (HR 0.778; 95\% CI 0.655-0.925) [27]. Orezzoli reported 5-year survival rates of $75 \%$ and $34 \%$ in two groups of patients with OCCC [28]. These values were in agreement with our results. However, no significant differences in survival rates between two groups of patients were observed by Scarfone [9] and Acién [29]. Multivariate analysis using a Cox regression model revealed that endometriosis remained being the independent impact factor affecting DFS, but not for OS. Besides, FIGO stage, receiving lymphadenectomy, residual disease and chemo-resistance were independent factors affecting the DFS rate in EOC patients. While, FIGO stage, residual disease, the courses of chemotherapy, chemo-resistance and hypertension were independent factors affecting the OS rate. These findings are partially in agreement with the results reported by Garrett et al. [17], Yang et al. [30] and Nasioudis et al. [31]. Collectively, the above findings suggest that the presence of EM might predict a better clinical prognosis for EOC patients. Our data also showed hypertension was the independent predictive factor for OS, which finding had been reported by Minlikeeva et al. [32]. The underlying mechanism of correlation between hypertensions and survival of EOC patients is unclear and need to be explored.

Admittedly, this study did not embrace all variable histotypes of EOC, but only included clear cell and endometrioid subtypes. Garrett et al. analyzed the prognosis of patients with different histological types of OC and observed a better prognosis in patients with endometrioid carcinoma [17]. As we have known, clear cell and endometrioid carcinoma occupied of the massive majority of EAOC, which were also indicated by our previous results. With this in mind, it was reasonable to obtain reliable information about the characteristics for EAOC by analyzing the data extracted from this series of patients.

\section{Conclusions}

This study showed that EOC patients with coexisting EM (EAOC group) were characterized by a younger age of onset, a higher percentage of premenopausal status, lower preoperative CA125 levels, an early tumor stage, a greater extent of satisfying tumor debulking, less opportunity of lymph node metastasis and developing chemoresistance. The EAOC patients also exhibited better survival, and EM itself was an independent factor affecting DFS. This study was a retrospective case analysis.
Certain biases might exist in the results due to inconsistency of the clinical features of the enrolled patients and lack of integrity of the collected clinical data. We expect that further research on this topic will be conducted, particularly at the level of molecular biology to facilitate the screening and early diagnosis of the population at high risk for malignant transformation of EM, ultimately improving the clinical prognosis of the disease.

\section{Abbreviations \\ CA-125: cancer antigen 125; DFS: Disease free survival; EAOC: Endometriosis- associated ovarian cancer; EM: Endometriosis; EOC: Epithelial ovarian carcinoma; FIGO: International Federation of Gynecology and Obstetrics; OCCC: Ovarian clear cell carcinoma; OEC: Ovarian endometrioid carcinoma; OS: Overall survival; ROC: Receiver Operating Characteristic}

\section{Acknowledgements}

We appreciate the gynecologists at Peking Union Medical College Hospital for their diligent clinical work and precise data recording about the cases we reported in this article.

\section{Funding}

This study was funded by National Natural Science Foundation of China (No. 81501236) and National Program on Key Research Project (No.SQ2017YFSF080001).

\section{Availability of data and materials}

The dataset supporting the conclusions of this article is included within the article and its additional files.

\section{Authors' contributions}

TR and SW make substantial contributions to conception and design, analysis and interpretation of data, and drafting the article. JS and JMQ have contributed to acquisition of data and analysis of data. YX and KS contribute to the valuable suggestion and consultation in preparing article. SW and JHL have designed the study and participate in revising it critically for important intellectual content. All authors read and approved the final manuscript.

Ethics approval and consent to participate

Peking Union Medical College Hospital Human Research Ethics Committee Approval was obtained for the use of all samples.

Consent for publication

Not applicable.

\section{Competing interests}

The authors declare that they have no competing interests.

\section{Publisher's Note}

Springer Nature remains neutral with regard to jurisdictional claims in published maps and institutional affiliations.

\footnotetext{
Author details

${ }^{1}$ Department of Obstetrics and Gynecology, Peking Union Medical College Hospital, Peking Union Medical College \& Chinese Academy of Medical Sciences, Shuaifuyuan 1, Wangfujing, Dongcheng District, Beijing 100730, People's Republic of China. ${ }^{2}$ Department of Pathology, Peking Union Medical College Hospital, Peking Union Medical College \& Chinese Academy of Medical Sciences, Beijing, People's Republic of China. ${ }^{3}$ School of Public Health, Peking Union Medical College \& Chinese Academy of Medical Sciences, Beijing, People's Republic of China.
} 
Received: 21 July 2017 Accepted: 25 September 2017

Published online: 03 October 2017

\section{References}

1. Sampson JA. Metastatic or embolic endometriosis, due to the menstrual dissemination of endometrial tissue into the venous circulation. Am J Pathol. 1927:3(2):93-110.

2. Scott RB. Malignant changes in endometriosis. Obstet Gynecol. 1953;2(3): 283-9.

3. Somigliana E, Vigano P, Parazzini F, Stoppelli S, Giambattista E, Vercellini $P$. Association between endometriosis and cancer: a comprehensive review and critical analysis of clinical and epidemiological evidence. Gynecol Oncol. 2006:101(2):331-41.

4. Viganò $P$, Somigliana E, Parazzini F, Vercellini P. Bias versus causality: interpreting recent evidence of association between endometriosis and ovarian cancer. Fertil Steril. 2006;88(3):588-93. 19

5. Melin A, Sparén P, Persson I, et al. Endometriosis and the risk of cancer with special emphasis on ovarian cancer. Hum Reprod. 2006;21:1237-42.

6. Kato M, Takano M, Miyamoto M, et al. Effect of ARID1A/BAF250a expression on carcinogenesis and clinicopathological factors in pure-type clear cell adenocarcinoma of the ovary. Mol Clin Oncol. 2016;5(4):395-401.

7. Fujiwara K, Shintani D, Nishikawa T. Clear-cell carcinoma of the ovary. Ann Oncol. 2016;27(Suppl 1):150-2.

8. Kaur S, Kerkar RA, Maheshwari A, et al. Clinical characteristics with patterns of relapse and survival analysis of ovarian clear cell carcinoma. Indian J Cancer. 2016:53(2):288-91.

9. Wang S, Qiu L, Lang JH, Shen K, Yang JX, Huang HF, et al. Clinical analysis of ovarian epithelial carcinoma with coexisting pelvic endometriosis. Am J Obstet Gynecol. 2013;208(413):e1-5.

10. Wang S, Qiu L, Lang JH, Shen K, Huang HF, Pan LY, Wu M, Yang JX, Guo LN. Prognostic analysis of endometrioid epithelial ovarian cancer with or without endometriosis: a 12-year cohort study of Chinese patients. Am J Obstet Gynecol. 2013;209(3):241.e1-9.

11. Lu J, Tao X, Zhou J, et al. Improved clinical outcomes of patients with ovarian carcinoma arising in endometriosis. Oncotarget. 2017:8(4):5843-52.

12. Morgan RJ Jr, Armstrong DK, Alvarez RD, Bakkum-Gamez JN, Behbakht K, Chen LM, et al. Ovarian Cancer, Version 1.2016, NCCN Clinical Practice Guidelines in Oncology. J Natl Compr Cancer Netw 2016;14(9):1134-1163.

13. Young RH, Scully RE. Metastatic tumors of the ovary. In: Kurman RJ, editor Blaustein's pathology of the female genital tract. New York: Springer; 1994 p. 1063-101.

14. Yang $Y H$, Chen RJ, Lin MC, Cheng SP, Chang TC. Synchronous primary ovarian and endometrial cancer with a fair prognosis in a young woman. Taiwan J Obstet Gynecol. 2010;49:97-100.

15. Ree YS, Cho SH, Kim SR, Cho SH, Kim KT, Park MH. Synchronous primary endometrial and ovarian cancer with three different histologic patterns: a case report. Int J Gynecol Cancer. 2003;13:678-82.

16. Nezhat F, Datta MS, Hanson V, Pejovic T, Nezhat C, Nezhat C. The relationship of endometriosis and ovarian malignancy: a review. Fertil Steril. 2008:90(5):1559-70.

17. Garrett LA, Growdon WB, Goodman A, Boruta DM, Schorge JO, del Carmen MG. Endometriosis-associated ovarian malignancy: a retrospective analysis of presentation, treatment, and outcome. J Reprod Med. 2013;58(11-12): 469-76.

18. Lim MC, Chun KC, Shin SJ, et al. Clinical presentation of endometrioid epithelial ovarian cancer with concurrent endometriosis: a multi-center retrospective study. Cancer Epidemiol Biomark Prev. 2010;19:398-404.

19. Scarfone G, Bergamini A, Noli S, et al. Characteristics of clear cell ovarian cancer arising from endometriosis: a two center cohort study. Gynecol Oncol. 2014:133:480-4.

20. Mangili G, Bergamini A, Taccagni G, Gentile C, Panina P, Viganò $P$, et al. Unravelling the two entities of endometrioid ovarian cancer: a single center clinical experience. Gynecol Oncol. 2012;126:403-7.

21. Bai H, Sha G, Xiao M, Gao H, Cao D, Yang J, Chen J, Wang Y, Zhang Z, Shen $K$. The prognostic value of pretreatment CA-125 levels and CA-125 normalization in ovarian clear cell carcinoma: a two-academic-institute study. Oncotarget 2016,29;7(13):15566-15576.

22. Munksgaard PS, Blaakaer J. The association between endometriosis and ovarian cancer: a review of histological, genetic and molecular alterations. Gynecol Oncol. 2012;124(1):164-9.
23. Van Gorp T, Amant F, Neven P, et al. Endometriosis and the development of malignant tumours of the pelvis. A review of literature. Best Pract Res Clin Obstet Gynaecol. 2004;18:349-71.

24. Nagle CM, Olsen CM, Webb PM, et al. Endometrioid and clear cell ovarian cancers: a comparative analysis of risk factors. Green AC; Australian Cancer Study Group; Australian Ovarian Cancer Study Group. Eur J Cancer. 2008; 44(16):2477-84

25. Rice MS, Murphy MA, Tworoger SS. Tubal ligation, hysterectomy and ovarian cancer: A meta-analysis. J Ovarian Res. 2012;5(1):13.

26. Rice MS, Murphy MA, Vitonis AF, et al. Tubal ligation, hysterectomy and epithelial ovarian cancer in the New England Case-control Study. Int J Cancer. 2013;133(10):2415-21.

27. Kim HS, Kim TH, Chung HH, Song YS. Risk and prognosis of ovarian cancer in women with endometriosis: a meta-analysis. Br J Cancer. 2014 Apr 2; 110(7):1878-90.

28. Orezzoli JP, Russell AH, Oliva E, et al. Prognostic implication of endometriosis in clear cell carcinoma of the ovary. Gynecol Oncol. 2008;110:336-44.

29. Acién P, Velasco I, Acién M, Capello C, Vela P. Epithelial Ovarian Cancers and Endometriosis. Gynecol Obstet Investig. 2015;79:126-35.

30. Yang B, Wang D, Chen $H$, Yang F. The association between endometriosis and survival outcomes of ovarian cancer: Evidence-based on a metaanalysis. Niger J Clin Pract. 2015:18(5):577-83.

31. Nasioudis D, Chapman-Davis E, Witkin SS, Holcomb K. Prognostic significance of lymphadenectomy and prevalence of lymph node metastasis in clinically-apparent stage I endometrioid and mucinous ovarian carcinoma. Gynecol Oncol. 2017:144(2):414-9.

32. Minlikeeva AN, Freudenheim JL, Eng KH, et al. History of Comorbidities and Survival of Ovarian Cancer Patients, Results from the Ovarian Cancer Association Consortium. Cancer Epidemiol Biomark Prev. 2017;26(9):1470-3.

\section{Submit your next manuscript to BioMed Central and we will help you at every step:}

- We accept pre-submission inquiries

- Our selector tool helps you to find the most relevant journal

- We provide round the clock customer support

- Convenient online submission

- Thorough peer review

- Inclusion in PubMed and all major indexing services

- Maximum visibility for your research

Submit your manuscript at www.biomedcentral.com/submit
C) Biomed Central 